\title{
Assessment of the reporting quality of randomised controlled trials of massage
}

\author{
Xuan Zhang ${ }^{1,2}$, Lin Zhang ${ }^{3}$, Weifeng Xiong ${ }^{4}$, Xihong Wang ${ }^{3}$, Xiaohan Zhou ${ }^{4}$, Chen Zhao ${ }^{5}$, Guihua Tian 6 , \\ Hongcai Shang ${ }^{6}$, Taixiang $\mathrm{Wu}^{7}$, Jiangxia Miao ${ }^{8}$ and Zhaoxiang Bian ${ }^{1,2^{*}}$ (])
}

\begin{abstract}
Objective: To assess the reporting quality of randomised controlled trials (RCTs) of massage, particularly whether necessary elements related to massage interventions were adequately reported.

Methods: A total of 8 electronic databases were systematically searched for massage RCTs published in English and Chinese from the date of their inception to June 22, 2020. Quality assessment was performed using three instruments, namely the CONSORT (Consolidated Standards of Reporting Trials) 2010 Checklist (37 items), the CONSORT Extension for NPT (Nonpharmacologic Treatments) 2017 checklist (18 items), and a self-designed massage-specific checklist (16 items) which included massage rationale, intervention and control group details. Descriptive statistics were additionally used to analyse the baseline characteristics of included trials.
\end{abstract}

Results: A total of 2,447 massage RCTs were identified, of which most (96.8\%) were distributed in China. For the completeness of CONSORT, NPT Extension, and massage-specific checklists, the average reporting percentages were $50 \%, 10 \%$ and $45 \%$, respectively. Of 68 assessed items in total (exclusion of 3 repeated items on intervention), 42 were poorly presented, including 18 CONSORT items, 15 NPT items, and 9 massage-specific items. Although the overall quality of reporting showed slightly improvement in articles published after 2010, the international (English) journals presented a higher score of the CONSORT and NPT items, while the Chinese journals were associated with the increased score of massage-specific items.

Conclusion: The quality of reporting of published massage RCTs is variable and in need of improvement. Reporting guideline "CONSORT extension for massage" should be developed.

Keywords: CONSORT Extension, Tuina, Massage, Nonpharmacologic treatment, Randomised controlled trials (RCTs), Reporting quality

\section{Background}

Massage therapy (MT), an ancient form of medical technique, can be defined as a systematic manipulation of soft tissues of the body for pain reduction or other

\footnotetext{
*Correspondence: bianzxiang@gmail.com

${ }^{1}$ Chinese Clinical Trial Registry (Hong Kong), Hong Kong Chinese

Medicine Clinical Study Centre, School of Chinese Medicine, Hong Kong Baptist University, 307 Room, Jockey Club School of Chinese Medicine

Building, 7 Baptist University Road, Kowloon Tong, Kowloon, Hong Kong, HKSAR, China

Full list of author information is available at the end of the article
}

therapeutic purposes [1]. Archaeological studies have unearthed evidence of massage dating back to around $2700 \mathrm{BC}$, making it the forerunner of all other forms of massage and body work that exist today, from shiatsu to osteopathy [2]. Indeed, massage seems to have been the primary form of healing throughout history in places such as ancient Greece, where Hippocrates (the father of modern medicine) described medicine as "being the art of rubbing" [3]. In China, the earliest record of massage was in the Shang dynasty (c. 1600 BC), when it was called "Anmo" (e.g., pressing and rubbing). The most popular term, however, for Chinese massage is "Tuina" original author(s) and the source, provide a link to the Creative Commons licence, and indicate if changes were made. The images or other third party material in this article are included in the article's Creative Commons licence, unless indicated otherwise in a credit line to the material. If material is not included in the article's Creative Commons licence and your intended use is not permitted by statutory regulation or exceeds the permitted use, you will need to obtain permission directly from the copyright holder. To view a copy of this licence, visit http://creativecommons.org/licenses/by/4.0/. The Creative Commons Public Domain Dedication waiver (http://creativeco mmons.org/publicdomain/zero/1.0/) applies to the data made available in this article, unless otherwise stated in a credit line to the data. 
(e.g., pushing and grasping), a term that appeared in the first specialised book of pediatric tuina published in the Ming dynasty (c. 1400) [4]. As a traditional intervention of Chinese medicine (CM), a wide range of technical manipulations, such as pulling, pressing, tapping, shaking, vibrating, kneading, rolling, gliding, and acupressure, are included in Tuina therapy for stimulating meridians, relaxing muscles, relieving pain and stretching joints [5]. Besides CM, there are different styles in other countries, such as Swedish massage, Thai massage, oil massage, and aromatherapy massage [6]. With the functions of boosting blood circulation, removing blockages, realigning the musculoskeletal structure, and enhancing the body's natural healing abilities, massage practice has been, and continues to be, a vital part of complementary and alternative medicine (CAM) treatments around the world [7].

Recently, there is an increasing number of clinical trials on massage. These studies suggested that MT has been notably effective in enhancing the growth of infants, increasing attentiveness, decreasing depression and aggression, alleviating motor problems, reducing pain, and enhancing immune function [8]. However, some scholars have indicated that the quality of available massage trials is not optimal due to flaws in reporting and methodology [9]. Incomplete reporting is one such shortcoming, which not only affects the efficacy evaluation of massage interventions, but also compromises the grades of research evidence, even misleading the clinical practice [10]. Generally, "reporting quality" is the basis for both peer reviewers' and readers' judgments of scientific merit, of truth, of accuracy, and of clinical applicability. However, "reporting quality" is a general term that needs to be translated into specific criteria covering study rationale, trial design, measurements and analysis. Massage trials involve aspects that do not present in other types of trials or studies. Specifically, for massage trials, details of the massage environment, patients' posture, media used for massage, the locations, force, frequency, and duration of massage, manipulations and procedures, treatment provider background, etc., are critical for trial assessment and replication. Although the Consolidated Standards of Reporting Trials (CONSORT) Statement and its extension for nonpharmacological treatments (NPT) provide researchers with clear reporting checklists for RCTs [11, 12], no previous study has assessed whether massage-specific details are sufficiently reported in the current massage RCTs; nor has any study identified what key information affecting the quality of massage trials should be described in the reports.

Therefore, in this review, the assessments of reporting quality of massage trials were not only based on the checklists of CONSORT 2010 and NPT Extension 2017, but also on a self-designed massage-specific checklist (e.g., the rationale of massage selected, details of manipulation and techniques, description of the treatment provider and control groups). The objectives of this study were as follows: (a) to summarize the general characteristics of published RCTs on massage; (b) to assess their reporting quality based on the standard CONSORT and NPT checklists; and (c) to evaluate whether necessary information related to massage interventions were adequately reported according to the massage-specific items.

\section{Methods}

\section{Inclusion and exclusion criteria}

This study included RCTs with massage intervention(s) published in English and Chinese up to 22 June 2020. We included a wide range of massage therapy, such as Chinese massage (Tuina), Swedish massage, Thai massage, Malay massage, ice massage, oil massage, infant massage, abdomen massage, and foot massage. Massage intervention(s) may have been administered alone or in combination with other interventions of conventional Western medicine or CAM. There were no limitations in the types of participants and controls in the included studies. Repeat publications, non-randomised or noncontrolled trials, non-massage interventional trials, comprehensive interventions not focused on massage treatment, study protocols, reviews, observational studies, case reports, abstracts or full-text reports not found, non-human studies, and non-English or non-Chinese language reports were excluded.

\section{Search strategy}

The following eight databases, EMBASE, MEDLINE, Evidence-Based Medicine (EBM) Reviews, Allied and Complementary Medicine (AMED), The China National Knowledge Infrastructure (CNKI), VIP database, Wanfang database, and WHO International Clinical Trials Registry Platform (including 17 registries updated in June 2020), were searched from the date of their inception to June 22, 2020. Languages were restricted to English and Chinese. The search terms were "massage", "Chinese medicine", "manipulative", "reflexion therapy", "physical contact", "randomised", "randomisation", "randomized", and "clinical trial", etc. The detailed search strategy is given in Additional file 1: Appendix S1.

\section{Screening}

The titles and abstracts of the records were independently screened by two authors (LZ and WFX) based on selection criteria, and the full texts of potentially suitable articles were retrieved for further assessment. Disagreements, if any, were resolved by discussion or consultation with the third author (XZ). 


\section{Data extraction}

Two authors (LZ and XZ) independently extracted details on a number of general characteristics of included studies, mainly involving publication years and journals, authors' information, type of studied diseases, interventions and outcomes, and study design (e.g., assignment, blinding and sample size). To ensure consistency in the data extraction process, criteria for the extraction items and typical examples were developed and used on twenty articles (e.g., ten English papers and ten Chinese papers) for testing, and then subsequently used to extract data from all included records. When there was any uncertainty regarding a particular article, the data extracted were checked and resolved by the third researcher (ZXB).

\section{Assessment of reporting quality}

The reporting quality of included trials was evaluated according to (1) the 37-item (including sub-items) of the CONSORT 2010 checklist; (2) the 18-item of the CONSORT Extension for Trials of Nonpharmacologic Treatments (NPTs) 2017 checklist; and (3) the 16-item of a self-designed massage-specific checklist. This massagerelated checklist was developed by three researchers (ZXB, GHT, and XZ) based on a discussion about (1) the key elements of massage in clinical practice; (2) transparent reporting of massage details in the clinical trials, such as the treatment posture, media used, location selected, manipulations and procedures, patients' responses, and adverse effects; (3) the rationale of why massage intervention(s) was selected; and (4) relevant items from the CONSORT Extensions for Chinese herbal medicine (CHM) formula, acupuncture, moxibustion, and cupping [13-16]. Aiming for easy calculation, the specifics of this checklist were categorized into six items with sixteen sub-questions, covering massage rationale, details of technique, treatment regimen, other components of treatment, massage provider background, and control or comparator of massage.

There is a total of 71 items (e.g., CONSORT 37-item, NPT 18-item, and massage-specific 16-item) in the above evaluation tools. As the massage-specific checklist was focused on an extension for the details of intervention, we thereby did not assess the three generalised items in the CONSORT and NPT Extension which with the same contents of the intervention, namely item 5 intervention of the CONSORT, and item $5 \mathrm{a}$ and $5 \mathrm{~b}$ of NPT Extension. Therefore, a total of 68 items (including CONSORT 36-item, NPT 16-item, and massage-specific 16-item) were actually assessed in this study. Each item/question was scored as "yes" if it was fully reported (Y, marked as 1 point) or "no" if it was incompletely reported or absent ( $\mathrm{N}$, marked as 0 point). The details of scoring rules are presented in Additional file 1: Appendix S2. Each included article was assessed by two researchers (LZ and $\mathrm{XZ}$ ) independently, and the results were double-checked. Any problems or ambiguities that arose were resolved by the third researcher $(\mathrm{ZXB})$.

\section{Statistical analysis}

For each assessed item, the number ( $\mathrm{n}$ ) and percentage (\%) of trials that completely reported the item were provided. The reporting percentages were arbitrarily categorized as excellent (>85\%), good (between 50\% and 85\%), and poor $(<50 \%)$. The mean overall CONSORT and NPT scores (e.g., the full score is 52), and massage-specific scores (e.g., the full score is 16) were calculated by subgroups and recorded as mean and standard deviations (SD). As indicated in the previous studies, the development of reporting guideline is closely related to the reporting quality of clinical trials [17]. Some scholars have identified that the publish year of guideline and types of journals (e.g., various in the reporting requirements and endorsement degree of the guideline) are the most essential and direct factors related to reporting quality $[18,19]$. Therefore, the selection of subgroups focused on (1) Year group, and (2) Journal group. Further, as the CONSORT 2010 guideline is the standard reporting framework for the RCTs, the Year group was separated as before (19902010) and after (2011-2020) the year of 2010. Regarding different types of journals, the major difference of reporting requirements is commonly existing between Chinese journals and international journals [20,21]. Based on the language selection in this study, the Journal group was defined as the "Chinese journal" and the "International (English) journal”. All data were collected and recorded in Microsoft Office Excel (Version 2016). Descriptive statistics were performed using SPSS software, version 25.0.

\section{Results \\ Search}

The initial search identified a total of 12,851 records, of which 8,691 relevant studies were retained after excluding duplicates and screening the titles and abstracts. After reviewing the full text of these articles, we identified 2,447 RCTs of massage for inclusion in our final analysis (Fig. 1).

\section{Characteristics of included trials}

The earliest RCT of massage included in this study was published in 1990. Since 2011, the number of trials has increased markedly, and more than half (57.8\%) were published during 2017-2019 (Fig. 2). All 2,447 trials were distributed in 15 countries, of which China makes the greatest contribution to the number of massage RCTs. Among 19 reported types of massage interventions, 
Records identified through

Chinese databases (CNKI, VIP

and Wanfang) searching:

( $n=11,014$ )
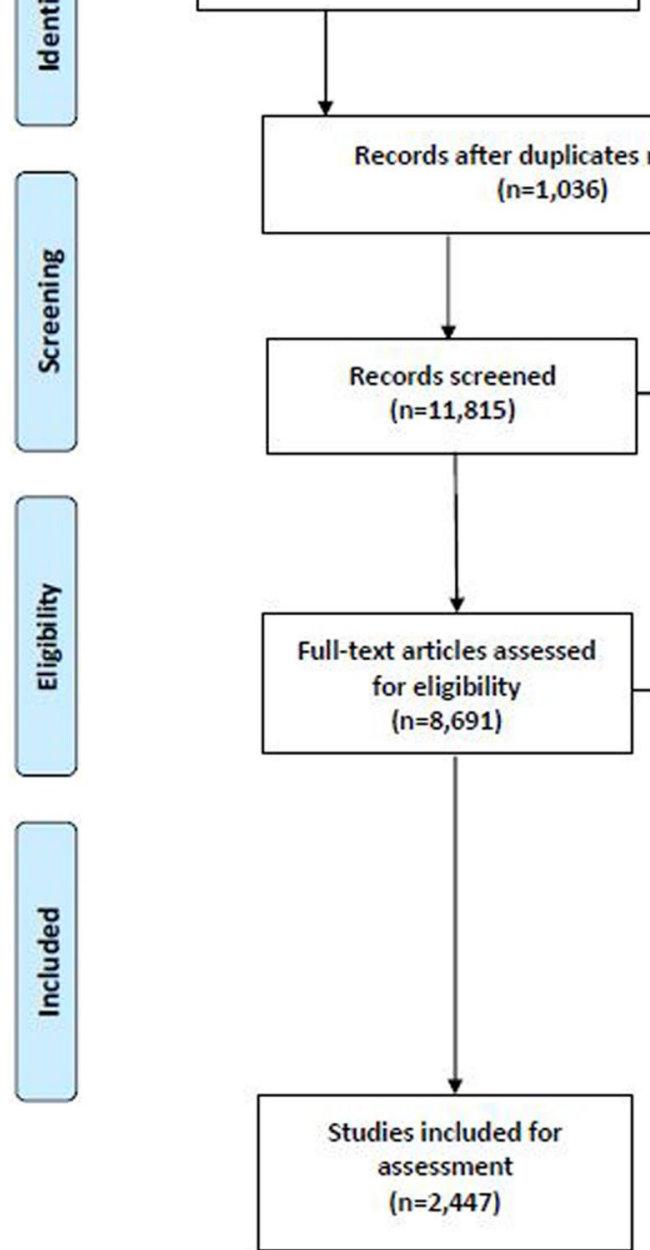

Records excluded after screening the title and abstracts $(n=3,124)$

Full-text articles excluded, with reasons ( $n=6,244)$

-Non-randomised trial $(n=574)$

-Non-controlled trial $(n=141)$

-SR/Meta $(n=175)$

-Review ( $n=715$ )

-Observational study $(n=36)$

- Case report $(n=13)$

-Non-English/Chinese language study $(n=10)$

-Non-human study ( $n=62$ )

-Study protocol $(n=5)$

-Abstract without full-text ( $n=37$ )

-No massage intervention included $(n=4,476)$,

including Western medicine

(pharmacological: $n=51$, and non-

pharmacological: $n=373$ ); CAM (herbal

medicine: $n=158$, acupoint injection: $n=720$,

acupoint application of herbal medicines:

$n=1,190$, and acupuncture, moxibustion and

cupping therapy: $n=1,757$ ); and

comprehensive interventions $(n=227)$

Fig. 1 Flow chart of the search and selection process

Chinese massage (also called Tuina which is based on the CM theory) was identified as the most common style (Fig. 3).

Most of the included trials (96.8\%) were published in Chinese journals, of these journals, only $29.6 \%$ were classified as medical core journals. The number of authors was mainly $1-5(87.5 \%)$, but $36.8 \%$ of articles reported only one author. The most common condition studied is the disease of musculoskeletal system or connective tissue $(44.1 \%)$. Commonly, massage RCTs were performed in a single centre (97.6\%), with a sample size of 51-100 (56.5\%), and with two assignment groups (95.7\%). Of 2,447 trials, $45.3 \%$ did not obtain or provide ethical approval, and $23.5 \%$ did not declare a conflict of interest. Few trials used the blinding methods (3.2\%) for massage interventions, and less than $15 \%$ of trials adopted the CM pattern (or other CM-related indicators) in the assessed conditions and outcomes. $72.1 \%$ of articles have been cited less than five times (Table 1).

\section{Reporting quality of included trials}

As for completeness in reporting, i.e., the number of checklist items reported in a given $\mathrm{RCT}$, the average reporting rates were $50 \%(0.2-99.8 \%)$ for CONSORT, $10 \%(0.04-57 \%)$ for NPT Extension, and 45\% (1.894.4\%) for massage-specific items, respectively. For all 68 

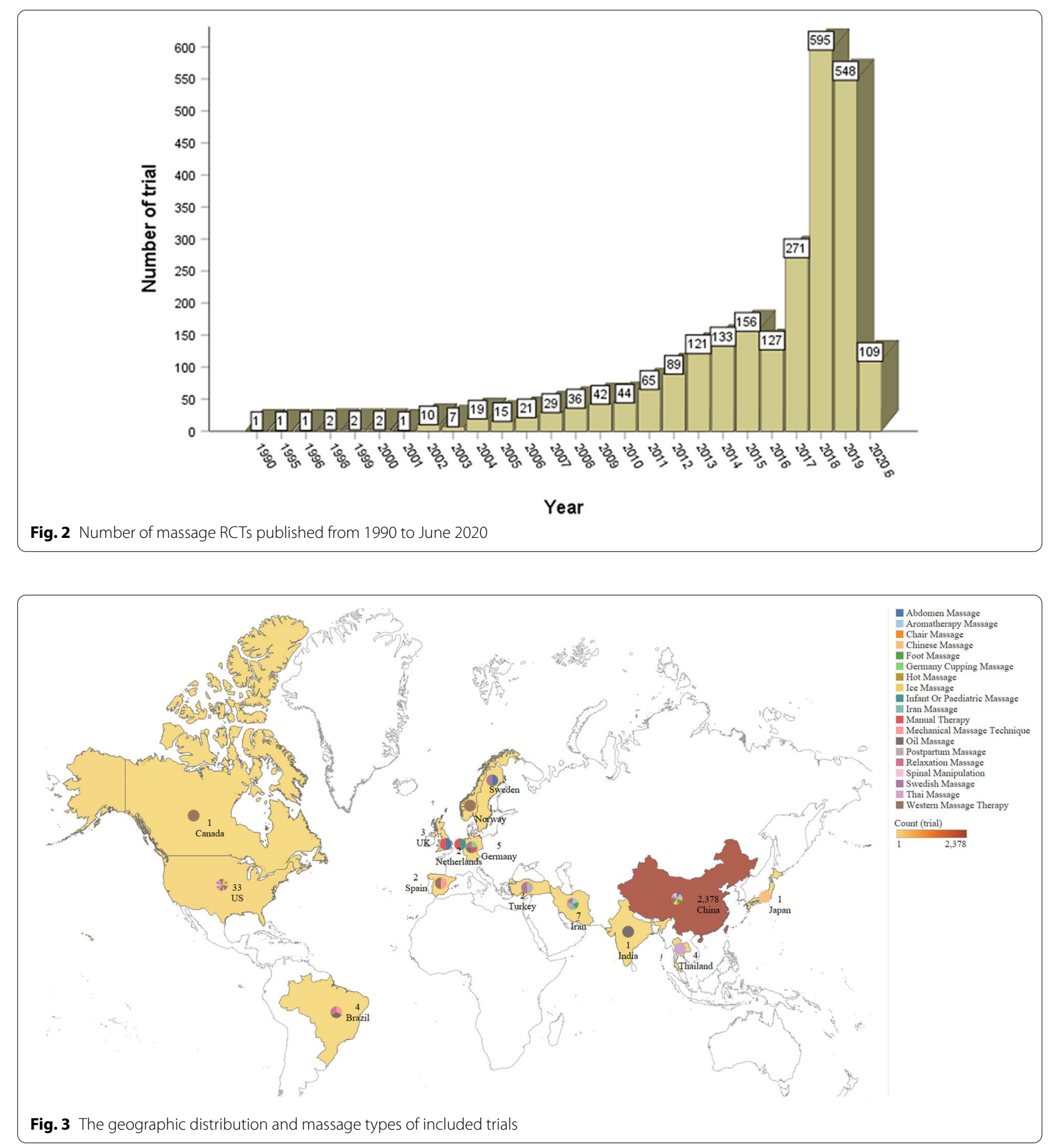

assessed items, reporting quality of $29 \%$ (20 items) was "excellent" (> 85\%) and of 9\% (6 items) was "good" (50 $85 \%)$; but $62 \%$ (42 items) were poorly reported $(<50 \%)$. Specifically, the poor reporting referred to 18 CONSORT items, 15 NPT items, and 9 massage-specific items. Details are provided in Tables 2 and 3.
In the subgroup of before and after the year of 2010, the overall reporting scores increased slightly for both CONSORT and NRT items, and massage-specific items from 19.6 (2.9) to 19.8 (2.8), out of 52 in total, and from 7.3 (1.9) to 7.6 (1.9), out of 16 in total. In the comparison of journals, the international (English) group showed 
Table 1. Characteristics of included articles $(n=2447)$

\begin{tabular}{|c|c|}
\hline Characteristic & $n(\%)$ \\
\hline \multicolumn{2}{|l|}{ Languages of published journals } \\
\hline Chinese & $2368(96.8)$ \\
\hline English & $79(3.2)$ \\
\hline \multicolumn{2}{|l|}{ Type of published journals } \\
\hline Comprehensive medical journals & $936(38.3)$ \\
\hline CAM/CM specific journals & $1288(52.6)$ \\
\hline Massage specific journals & $223(9.1)$ \\
\hline \multicolumn{2}{|l|}{ Grades of Chinese journals } \\
\hline Medical core journals $\mathrm{s}^{\mathrm{a}}$ (Yes) & $702(29.6)$ \\
\hline \multicolumn{2}{|l|}{ Impact factor of international (English) journals ${ }^{b}$} \\
\hline $0-4$ & $75(94.9)$ \\
\hline $5-10$ & $1(1.3)$ \\
\hline$>10$ & $3(3.8)$ \\
\hline \multicolumn{2}{|l|}{ Number of authors included } \\
\hline 1 & $901(36.8)$ \\
\hline $2-5$ & $1241(50.7)$ \\
\hline $6-10$ & $289(11.8)$ \\
\hline$>10$ & $16(0.7)$ \\
\hline \multicolumn{2}{|l|}{ Institution of the corresponding author } \\
\hline Hospital & $2279(93.1)$ \\
\hline University & $137(5.6)$ \\
\hline Research Institute & $28(1.1)$ \\
\hline Others & $3(0.1)$ \\
\hline \multicolumn{2}{|l|}{ Type of conditions treated (top five) } \\
\hline $\begin{array}{l}\text { Diseases of the musculoskeletal system or connective } \\
\text { tissue }\end{array}$ & $1,078(44.1)$ \\
\hline $\begin{array}{l}\text { Symptoms, signs or clinical findings, not elsewhere clas- } \\
\text { sified }\end{array}$ & $249(10.2)$ \\
\hline Diseases of the nervous system & $236(9.6)$ \\
\hline Pregnancy, childbirth or the puerperium & $180(7.4)$ \\
\hline Diseases of the digestive system & $174(7.1)$ \\
\hline \multicolumn{2}{|l|}{ Studied disease(s) with CM Pattern(s) } \\
\hline Yes & $155(6.3)$ \\
\hline \multicolumn{2}{|l|}{ Experimental group } \\
\hline Only massage intervention(s) & $664(27.1)$ \\
\hline Other interventions administered to massage & $1783(72.9)$ \\
\hline \multicolumn{2}{|l|}{ Type of blinding } \\
\hline Single & $60(2.5)$ \\
\hline Double & $16(0.7)$ \\
\hline Open label & $2,306(94.2)$ \\
\hline Not reported & $65(2.7)$ \\
\hline \multicolumn{2}{|l|}{ Number of assigned groups } \\
\hline 2 & $2342(95.7)$ \\
\hline 3 & $80(3.3)$ \\
\hline$>3$ & $25(1.0)$ \\
\hline \multicolumn{2}{|l|}{ Sample size } \\
\hline $1-50$ & $239(9.8)$ \\
\hline $51-100$ & $1383(56.5)$ \\
\hline $101-300$ & $764(31.2)$ \\
\hline $301-500$ & $38(1.6)$ \\
\hline
\end{tabular}

Table 1. (continued)

\begin{tabular}{lr}
\hline Characteristic & $\boldsymbol{n}$ (\%) \\
\hline$>500$ & $23(1.0)$ \\
Trial participating centre & $2388(97.6)$ \\
Single centre & $59(2.4)$ \\
Multi-centre & \\
Outcomes including CM-related indicators & $181(7.4)$ \\
Yes & \\
Number of times cited & $566(23.1)$ \\
0 & $1200(49.0)$ \\
$1-5$ & $339(13.9)$ \\
$6-10$ & $229(9.4)$ \\
$11-20$ & $113(4.6)$ \\
$>20$ & \\
Ethics approval & $1339(54.7)$ \\
Yes & \\
Declaration of conflict of interest & $1871(76.5)$ \\
Yes
\end{tabular}

CAM complementary and alternative medicine, $C M$ Chinese medicine

${ }^{\text {a }}$ According to the 2019 China Science and Technology Core Journals Catalog (Natural Science Volume) [22]

${ }^{b}$ The impact factor (IF) of an academic international journal is a scientometric index calculated by Clarivate that reflects the yearly average number of citations of articles published in the last two years in a given journal. The record of IF was collected from the journal formal website of each targeted journal in Oct 2020.

a higher score of 22.7 (3.7) in the CONSORT and NRT items, and the Chinese journals associated with the increased score of 7.6 (1.9) in the massage-specific items (Table 4).

\section{Discussion}

This study provides a literature review of the reporting characteristics and quality of massage RCTs in the past 30 years, which have identified a remarkable increase in the number of publications since 2017. Although RCT is regarded as the gold standard among different types of clinical studies, the quality of massage RCTs included in this review appears to be far from optimal. Incomplete or absent reporting of key trial information leads to some difficulties for assessing the quality of the whole study, easily inviting skepticism as to its results. Similar problems were also found in the previous study examining systematic reviews of massage treatments [10].

In this study, we found several problems in the characteristics of articles reporting massage RCTs. First, few studies were published in relatively high-quality journals. For the $96.8 \%$ of trials published in Chinese journals, only 702 articles were published in the "Chinese Medical Core Journals" [22]. For the remaining 3.2\% published in international (English) journals, only 4 articles were rated as having a relatively high-impact factor (e.g., IF > 
Table 2. Reporting quality of 2,447 included trials based on the CONSORT and CONSORT for NPT items

\begin{tabular}{|c|c|c|}
\hline Section/topic & Item number and description & Item present, $\mathrm{n}(\%)$ \\
\hline \multirow[t]{4}{*}{ Title and abstract } & 1a. Identification as a randomised trial in the title & $656(26.8)$ \\
\hline & $\begin{array}{l}\text { 1b. Structured summary of trial design, methods, results, and conclusions (for specific } \\
\text { guidance see CONSORT for abstracts) }\end{array}$ & $2420(98.9)$ \\
\hline & $\begin{array}{l}\text { 16 NPT. When applicable, report eligibility criteria for centers where the intervention is per- } \\
\text { formed and for care providers }\end{array}$ & $1395(57.0)$ \\
\hline & Ib NPT. Report any important changes to the intervention delivered from what was planned & $543(22.2)$ \\
\hline \multicolumn{3}{|l|}{ Introduction } \\
\hline \multirow[t]{2}{*}{ Background and objectives } & 2a. Scientific background and explanation of rationale & $2364(96.7)$ \\
\hline & 2b. Specific objectives or hypotheses & $2395(97.9)$ \\
\hline \multicolumn{3}{|l|}{ Methods } \\
\hline \multirow[t]{3}{*}{ Trial design } & 3a. Description of trial design (such as parallel, factorial) including allocation ratio & $77(3.1)$ \\
\hline & 3a NPT. When applicable, how care providers were allocated to each trial group & $945(38.6)$ \\
\hline & $\begin{array}{l}\text { 3b. Important changes to methods after trial commencement (such as eligibility criteria), } \\
\text { with reasons }\end{array}$ & $1676(68.5)$ \\
\hline \multirow[t]{3}{*}{ Participants } & 4a. Eligibility criteria for participants & $2400(98.1)$ \\
\hline & 4a NPT. When applicable, eligibility criteria for centers and for care providers & $52(2.1)$ \\
\hline & 4b. Settings and locations where the data were collected & $2431(99.3)$ \\
\hline \multirow[t]{6}{*}{ Interventions } & $\begin{array}{l}\text { 5. Interventions for each group with sufficient details to allow replication, including how } \\
\text { and when they were actually administered }\end{array}$ & See Table 3 \\
\hline & 5a NPT. Precise details of both the experimental treatment and comparator & See Table 3 \\
\hline & $\begin{array}{l}56 \text { NPT. Description of the different components of the interventions and, when applicable, } \\
\text { description of the procedure for tailoring the interventions to individual participants. }\end{array}$ & See Table 3 \\
\hline & 5c NPT. Details of whether and how the interventions were standardized. & $943(38.5)$ \\
\hline & $\begin{array}{l}5 d \text { NPT. Details of whether and how adherence of care providers to the protocol was assessed } \\
\text { or enhanced }\end{array}$ & $121(4.9)$ \\
\hline & $\begin{array}{l}\text { 5e NPT. Details of whether and how adherence of participants to interventions was assessed } \\
\text { or enhanced }\end{array}$ & $7(0.3)$ \\
\hline \multirow[t]{2}{*}{ Outcomes } & $\begin{array}{l}\text { 6a. Completely defined pre-specified primary and secondary outcome measures, includ- } \\
\text { ing how and when they were assessed }\end{array}$ & $1431(58.5)$ \\
\hline & 6b. Any changes to trial outcomes after the trial commenced, with reasons & $4(0.2)$ \\
\hline \multirow[t]{3}{*}{ Sample size } & 7a. How sample size was determined & $844(34.5)$ \\
\hline & $\begin{array}{l}\text { 7a NPT. When applicable, details of whether and how the clustering by care providers or cent- } \\
\text { ers was addressed }\end{array}$ & $3(0.1)$ \\
\hline & 7b. When applicable, explanation of any interim analyses and stopping guidelines & $10(0.4)$ \\
\hline \multirow[t]{2}{*}{ Sequence generation } & 8a. Method used to generate the random allocation sequence & $652(26.6)$ \\
\hline & 8b. Type of randomisation; details of any restriction (such as blocking and block size) & $78(3.2)$ \\
\hline Allocation concealment mechanism & $\begin{array}{l}\text { 9. Mechanism used to implement the random allocation sequence (such as sequentially } \\
\text { numbered containers), describing any steps taken to conceal the sequence until } \\
\text { interventions were assigned }\end{array}$ & $74(3.0)$ \\
\hline Implementation & $\begin{array}{l}\text { 10. Who generated the random allocation sequence, who enrolled participants, and who } \\
\text { assigned participants to interventions }\end{array}$ & $46(1.9)$ \\
\hline \multirow[t]{4}{*}{ Blinding } & $\begin{array}{l}\text { 11a. If done, who was blinded after assignment to interventions (for example, partici- } \\
\text { pants, care providers, those assessing outcomes) and how }\end{array}$ & $2343(95.7)$ \\
\hline & $\begin{array}{l}11 \text { a NPT. If done, who was blinded after assignment to interventions (e.g., participants, care } \\
\text { providers, those administering co-interventions, those assessing outcomes) and how }\end{array}$ & $27(1.1)$ \\
\hline & 11b. If relevant, description of the similarity of interventions & $23(0.9)$ \\
\hline & 11c NPT. If blinding was not possible, description of any attempts to limit bias & $12(0.5)$ \\
\hline \multirow[t]{3}{*}{ Statistical methods } & 12a. Statistical methods used to compare groups for primary and secondary outcomes & $2373(97.0)$ \\
\hline & $\begin{array}{l}\text { 12a NPT. When applicable, details of whether and how the clustering by care providers or } \\
\text { centers was addressed }\end{array}$ & $1(0.04)$ \\
\hline & 12b. Methods for additional analyses, such as subgroup analyses and adjusted analyses & $10(0.4)$ \\
\hline
\end{tabular}


Table 2. (continued)

\begin{tabular}{|c|c|c|}
\hline Section/topic & Item number and description & Item present, $\mathrm{n}(\%)$ \\
\hline \multirow[t]{4}{*}{$\begin{array}{l}\text { Participant flow (a diagram is } \\
\text { strongly recommended) }\end{array}$} & $\begin{array}{l}\text { 13a. For each group, the numbers of participants who were randomly assigned, received } \\
\text { intended treatment, and were analysed for the primary outcome }\end{array}$ & $2440(99.7)$ \\
\hline & $\begin{array}{l}\text { 13a NPT. The number of care providers or centers performing the intervention in each group } \\
\text { and the number of patients treated by each care provider or in each center }\end{array}$ & $3(0.1)$ \\
\hline & 13b. For each group, losses and exclusions after randomisation, together with reasons & $2441(99.8)$ \\
\hline & $\begin{array}{l}\text { 13c NPT. For each group, the delay between randomization and the initiation of the interven- } \\
\text { tion }\end{array}$ & $2(0.1)$ \\
\hline \multirow[t]{2}{*}{ Recruitment } & 14a. Dates defining the periods of recruitment and follow-up & $2376(97.1)$ \\
\hline & 14b. Why the trial ended or was stopped & $2367(96.7)$ \\
\hline \multirow[t]{2}{*}{ Baseline data } & 15. A table showing baseline demographic and clinical characteristics for each group & $324(13.2)$ \\
\hline & $\begin{array}{l}15 \text { NPT. When applicable, a description of care providers (case volume, qualification, expertise, } \\
\text { etc.) and centers (volume) in each group }\end{array}$ & $4(0.2)$ \\
\hline Numbers analysed & $\begin{array}{l}\text { 16. For each group, number of participants (denominator) included in each analysis and } \\
\text { whether the analysis was by original assigned groups }\end{array}$ & $2336(95.5)$ \\
\hline \multirow[t]{2}{*}{ Outcomes and estimation } & $\begin{array}{l}\text { 17a. For each primary and secondary outcome, results for each group, and the estimated } \\
\text { effect size and its precision (such as } 95 \% \text { confidence interval) }\end{array}$ & $2319(94.8)$ \\
\hline & $\begin{array}{l}\text { 17b. For binary outcomes, presentation of both absolute and relative effect sizes is } \\
\text { recommended }\end{array}$ & $2183(89.2)$ \\
\hline Ancillary analyses & $\begin{array}{l}\text { 18. Results of any other analyses performed, including subgroup analyses and adjusted } \\
\text { analyses, distinguishing pre-specified from exploratory }\end{array}$ & $8(0.3)$ \\
\hline Harms & $\begin{array}{l}\text { 19. All important harms or unintended effects in each group (for specific guidance see } \\
\text { CONSORT for harms) }\end{array}$ & $148(6.0)$ \\
\hline \multicolumn{3}{|l|}{ Discussion } \\
\hline \multirow[t]{2}{*}{ Limitations } & $\begin{array}{l}\text { 20. Trial limitations, addressing sources of potential bias, imprecision, and, if relevant, } \\
\text { multiplicity of analyses }\end{array}$ & $117(4.8)$ \\
\hline & $\begin{array}{l}20 \text { NPT. In addition, take into account the choice of the comparator, lack of or partial blinding, } \\
\text { and unequal expertise of care providers or centers in each group }\end{array}$ & $10(0.4)$ \\
\hline \multirow[t]{2}{*}{ Generalisability } & 21. Generalisability (external validity, applicability) of the trial findings & $2185(89.3)$ \\
\hline & $\begin{array}{l}21 \text { NPT. Generalizability (external validity) of the trial findings according to the intervention, } \\
\text { comparators, patients, and care providers and centers involved in the trial }\end{array}$ & $3(0.1)$ \\
\hline Interpretation & $\begin{array}{l}\text { 22. Interpretation consistent with results, balancing benefits and harms, and considering } \\
\text { other relevant evidence }\end{array}$ & $2308(94.3)$ \\
\hline \multicolumn{3}{|l|}{ Other information } \\
\hline Registration & 23. Registration number and name of trial registry & $57(2.3)$ \\
\hline Protocol & 24. Where the full trial protocol can be accessed, if available & $38(1.6)$ \\
\hline Funding & 25. Sources of funding and other support (such as supply of drugs), role of funders & $495(20.2)$ \\
\hline
\end{tabular}

5). Higher-quality journals usually have broader reviewer networks and more rigorous reporting requirements. Previous studies have indicated that the reporting quality of clinical trials is positively correlated with journal quality [19]. Secondly, CM-related indicators were rarely mentioned in the design of massage RCTs. Although CM-based massage intervention (e.g., Tuina/Chinese massage) was the most commonly used, less than $15 \%$ of trials adopted CM pattern as diagnostic criteria and outcome(s) for evaluation. Previous studies have pointed out that if a CM trial does not consider CM pattern identification ('zheng') or use CM 'zheng'-related outcome(s), participants may not be properly treated and/or the efficacy of CM intervention(s) may not be assessed properly [23]. Thirdly, the common study design was a two-arm-in-parallel group, with 51-100 sample size, open label and a single centre trial. Thus, high-quality massage RCTs with a large sample size and multicentre design are urgently needed. Blinding is not easily implemented in massage interventional RCTs, especially for doubleblind. In this study, we identified a total of 16 trials that reported a double-blind method. Except for 10 trials (e.g., 4 Chinese journal papers and 6 international journal papers) which defined the blinded people as subjects and outcome assessors/statisticians, the remaining 6 trials (e.g., 1 Chinese journal paper and 5 international journal papers) actually blinded both subjects and treatment providers. Among these 6 trials, 2 used oil massage (e.g., oil placebo), 1 adopted sham massage device (e.g., the same mechanical massage technique but with different 
Table 3. Assessment on the self-designed massage-specific checklist

\begin{tabular}{|c|c|c|}
\hline Item & Specifics & Yes, n (\%) \\
\hline \multirow[t]{3}{*}{ Massage rationale } & Q1. Whether the type of massage was reported? & $1504(61.5)$ \\
\hline & Q2. Whether the rationale for selected massage was provided? & $975(39.8)$ \\
\hline & Q3. Whether any information about individualized massage treatment was reported? & $82(3.4)$ \\
\hline \multirow[t]{7}{*}{ Details of massage } & Q4. Whether the patient posture and/or environment during treatment was mentioned? & $1697(69.4)$ \\
\hline & Q5. Whether the media (e.g., dosage and manufacturers) used for massage was reported? & $351(14.3)$ \\
\hline & Q6. Whether the massage points and/or locations were provided? & $2311(94.4)$ \\
\hline & $\begin{array}{l}\text { Q7. Whether the duration, frequency and/or force of massage in per point and/or location was } \\
\text { reported? }\end{array}$ & $2088(85.3)$ \\
\hline & Q8. Whether the details of procedure and technique of massage was described? & $1877(76.7)$ \\
\hline & Q9. Whether any responses sought of patients during/after massage was reported? & $690(28.2)$ \\
\hline & Q10. Whether any measures or management for possible adverse events was pre-mentioned? & $45(1.8)$ \\
\hline Treatment regimen & $\begin{array}{l}\text { Q11. Whether the number, frequency, duration and/or force of provided massage sessions was } \\
\text { reported? }\end{array}$ & $2297(93.9)$ \\
\hline \multirow[t]{2}{*}{ Other components of treatment } & $\begin{array}{l}\text { Q12. For complex interventions, whether the details of other interventions administered to the } \\
\text { massage group were reported? }\end{array}$ & $2159(88.2)$ \\
\hline & $\begin{array}{l}\text { Q13. Whether any instruction and/or information of selected massage was presented to treatment } \\
\text { providers and the participants? }\end{array}$ & $83(3.4)$ \\
\hline Treatment provider background & $\begin{array}{l}\text { Q14. Whether any description of treatment providers' background (e.g., qualification and/or experi- } \\
\text { ences in massage) was reported? }\end{array}$ & $149(6.1)$ \\
\hline \multirow[t]{2}{*}{ Control or comparator interventions } & Q15. Whether the rationale for the choice of the control(s) was provided? & $73(3.0)$ \\
\hline & Q16. Whether the details of control or comparator were described? & $1214(49.6)$ \\
\hline
\end{tabular}

Table 4. Overall reporting quality scores for 2,447 included trials, by subgroup

\begin{tabular}{|c|c|c|}
\hline \multirow[t]{2}{*}{ Subgroup } & \multicolumn{2}{|c|}{ For massage-specific items } \\
\hline & Mean (SD) score ${ }^{a}$ & Mean (SD) score \\
\hline \multicolumn{3}{|l|}{ Year of publication (n) } \\
\hline $1990-2010(n=233)$ & $19.6(2.9)$ & $7.3(1.9)$ \\
\hline $2011-2020(n=2,214)$ & $19.8(2.8)$ & $7.6(1.9)$ \\
\hline \multicolumn{3}{|l|}{ Journal of publication (n) } \\
\hline Chinese journal $(n=2368)$ & $20.1(2.6)$ & $7.6(1.9)$ \\
\hline International (English) journal $(n=79)$ & $22.7(3.7)$ & $5.5(1.5)$ \\
\hline
\end{tabular}

SD standard deviation

${ }^{\text {a }} \mathrm{A}$ perfect score is 52 for the CONSORT and NPT items

${ }^{\mathrm{b}} \mathrm{A}$ perfect score is 16 for the massage-specific items

settings of actual massage and sham massage), and 3 achieved sham control through coded different acupoints and locations. The treatment providers in these trials did not know which massage protocol is the placebo control. Finally, nearly half of the included trials did not report any information about ethical approval. Readers thereby cannot fully evaluate such researches as ethical declarations form a vital integral part of clinical trial [24].

The reporting quality of massage RCTs was assessed by three instruments in this review, including the CONSORT checklist, the CONSORT Extension for NPT checklist, and a self-designed massage-specific checklist.
Our checklist of message-specific information was designed to identify the critical elements in the rationale for choosing massage as a therapy, the details of massage techniques, and the design of a control for massage interventions. The overall quality of reporting has been unsatisfactory, although a slight improvement has been seen after the CONSORT was updated and issued in 2010.

With regard to the completeness of the CONSORT items, the average reporting rate was 50\% (ranging from 0.2 to $99.8 \%)$. 18 items were reported poorly $(<50 \%)$, of which most $(56 \%, 10 / 18)$ related to key methodological domains, such as trial design (item $3 a$ ), sample size 
(items $7 \mathrm{a}$ and $7 \mathrm{~b}$ ), sequence generation (items $8 \mathrm{a}$ and $8 \mathrm{~b}$ ), allocation concealment mechanism (item 9), implementation (item 10), blinding (item 11b), and additional analyses (item 12b). Previous studies have indicated that the methodology-related items are crucial for the assessment of bias risk and of the reliability of reported effects [2527]. Other items, including baseline data (item 15), ancillary analyses (item 18), harms (item 19), limitations (item 20 ), registration (item 23), protocol (item 24), and funding (item 25) in the "Results" and "Other information" sections, were also reported poorly. Inadequate reporting of trial registration, protocol, and ethical approval significantly compromises the value of massage RCTs, inviting skepticism and criticism [28].

At the same time, we identified a considerable number of NPT Extension items that were missing or incompletely reported; the average reporting rate was only $10 \%$, with a range of $0.04 \%$ to $57 \%$. Nonpharmacological treatments frequently involve multicomponent interventions delivered by multiple care providers, and each component or provider may influence the success of the overall intervention. Even though the CONSORT NPT Extension was developed in 2008 and updated in 2017 [12], the completeness of reporting remains insufficient, especially in terms of (1) adherence of participants to interventions; (2) changes to the intervention delivered from what was planned; (3) information about care providers; and (4) clustering by care providers or centers. Similar to previous studies, space constraints in journals, such as Chinese journals without an online appendix, is one reason for inadequate reporting of interventions in the RCTs of massage [29]. In this study, we also identified that the reporting score of the CONSORT and NPT items was lower in Chinese articles than that in the international (English) articles. Together, authors, editors (especially for Chinese journals), and reviewers should be more rigorous in their demands that authors adhere to the reporting standards of CONSORT and NPT items in trials of nonpharmacological treatments.

For massage- specific items, the average reporting rate was $45 \%$, with a range of $1.8 \%$ to $94.4 \%$. The least well reported information about massage interventions can be summarised in the following aspects: (1) the rationale of why the massage was being used and why the comparison was selected; (2) some details of procedure, particular in individualised massage treatments, media used (if any), responses sought of patients, and management for adverse events; and (3) the background of treatment provider(s). In practice, massage is very much practitioner and experience-dependent, thus, precise and complete details of the manipulation and related factors are essential for the transparency and replication of trials. Some previous analyses suggested that "post-2010 publication years" can be used as an independent predictor of the high reporting quality of RCTs [30], but no great improvement was identified in the included massage RCTs. In this study, we found the reporting score of massage-specific items in Chinese journals was better than that in international (English) journals. We surmise that this is because CM practitioners have a richer experience in conducting complex massage therapies and greater motivation to describe the technique details [31]. Much of traditional CM, since the Shang Dynasty, has been and is based on doctors recording their treatments. Modern doctors have read these earlier accounts, develop different Tuina/ Chinese massage schools, know the value of techniques inheritance, and hence are more likely to record those details from their own practice [32].

Given the deficiencies of reporting identified in this study, specific improvements are needed. Previous findings confirm that guidelines do help improve the quality of reporting $[17,33]$. Therefore, there are two paths forward: either strengthen reporting of the CONSORT and NPT Extension guidelines, or develop a series of standard reporting items specifically relevant to RCTs with massage interventions as another independent Extension to the general CONSORT 2010 statement. Taking the second route, our working group has initiated and registered the "STandards for Reporting Interventions in Clinical Trials Of Tuina/Massage (STRICTOTM): Extending the CONSORT Statement" on the EQUATOR (Enhancing the QUAlity and Transparency Of health Research) Network [34]. We intend to complete this reporting guideline at the end of 2021.

This study has some limitations. First, this review identified massage RCTs published up to 22 June 2020 in the targeted eight databases. Any records which had not been included in these databases by that cutoff period have not been included. In addition, we included only articles in English and Chinese because of language limitations. As such, we may not have captured otherwise eligible trials published in other languages. Second, we assessed each item with a "1" or " 0 " score according to whether the author had reported the detailed information listed in the proposed items. All incomplete reporting (e.g., partial and absent) was given as "0"; and some "not applicable" reporting (e.g., open-label without blinding) was categorized as "1". This over-simplifies the actual situation. Third, we did not assess the methodology quality (e.g., using a Cochrane tool) of each included trial as the primary objective of this study focused on the reporting characteristics. Although the results of this review may not necessarily be comprehensive, we do believe that the general trends indicated by this study are valid. 


\section{Conclusion}

Without complete and transparent reporting of how a massage RCT was designed and implemented, it is difficult for readers to assess the reliability and validity of trial findings. Although the CONSORT Statement and NPT Extension are key tools through which adequate reporting can be achieved, the quality of massage RCTs still needs improvement in terms of meeting quality standards and sufficient reporting of massage-specific information. Development of the reporting guideline for massage RCTs, as an extension of CONSORT, should be an effective strategy to improve the current situation.

\section{Abbreviations}

RCTs: Randomised controlled trials; CONSORT: Consolidated Standards of Reporting Trials; NPT: Nonpharmacologic treatments; MT: Massage therapy; CM: Chinese medicine; CAM: Complementary and alternative medicine; EBM: Evidence-based medicine; AMED: Allied and Complementary Medicine; CNKI: The China National Knowledge Infrastructure; WHO ICTRP: World Health Origination International Clinical Trials Registry Platform; CHM: Chinese herbal medicine; SD: Standard deviations; EQUATOR: Enhancing the QUAlity and Transparency Of health Research; IF: Impact factor.

\section{Supplementary Information}

The online version contains supplementary material available at https://doi. org/10.1186/s13020-021-00475-6.

Additional file 1. Search strategy. S2. Quality assessment rules of included trials.

\section{Acknowledgements}

The authors thank Dr. Martha Dahlen for providing valuable comments of the manuscript.

\section{Authors' contributions}

$Z X B$ and $X Z$ designed and drafted this article; $X Z, L Z$ and WFX searched and screened the articles; $Z X B, X Z$ and GHT developed the massage-specific checklist; XZ, LZ, WFX, XHW, XHZ and CZ assessed and analysed the data; HCS, TXW and JXM provided critical comments for the article; XZ and ZXB revised and finalized the manuscript. All authors read and approved the final manuscript.

\section{Funding}

This work was supported by the Chinese Medicine Development Fund, Hong Kong (No. 19B2/044A_R1). The funder had no role in the design of the study, in the collection, analysis, and interpretation of data, nor in the writing of the manuscript.

\section{Availability of data and materials}

The data used for this study are included in the manuscript and supplementary file.

\section{Declarations}

Ethics approval and consent to participate Not applicable.

\section{Consent for publication}

Not applicable.

\section{Competing interests}

The authors declare that they have no competing interests.

\section{Author details}

${ }^{1}$ Chinese Clinical Trial Registry (Hong Kong), Hong Kong Chinese Medicine Clinical Study Centre, School of Chinese Medicine, Hong Kong Baptist University, 307 Room, Jockey Club School of Chinese Medicine Building, 7 Baptist University Road, Kowloon Tong, Kowloon, Hong Kong, HKSAR, China. ${ }^{2}$ Chinese EQUATOR Centre, Hong Kong Baptist University, Hong Kong, HKSAR, China. ${ }^{3}$ Tianjin University of Traditional Chinese Medicine, Tianjin, China. ${ }^{4}$ College of Chinese Medicine, Beijing University of Chinese Medicine, Beijing, China. ${ }^{5}$ Institute of Basic Research in Clinical Medicine, China Academy of Chinese Medical Sciences, Beijing, China. ${ }^{6}$ Key Laboratory of Chinese Internal Medicine of Ministry of Education and Beijing, Dongzhimen Hospital, Beijing University of Chinese Medicine, Beijing, China. ${ }^{7}$ Chinese Cochrane Centre, West China Hospital, Sichuan University, China Trial Registration Center, Chengdu, Sichuan, China. ${ }^{8}$ School of Chinese medicine, The Chinese University of Hong Kong, Hong Kong, HKSAR, China.

Received: 3 June 2021 Accepted: 19 July 2021

Published online: 28 July 2021

\section{References}

1. Kennedy AB, Cambron JA, Sharpe PA, Travillian RS, Saunders RP. Clarifying definitions for the massage therapy profession: the results of the best practices symposium. IJTMB. 2016;9(3):15-26.

2. UK Register of Tui na Chinese Massage. History of Tui na Chinese Massage. http://www.ukrtcm.org/about-tui-na/history. Accessed 12 Nov 2020.

3. Field T. Massage therapy Complement Altern Med. 2002;86(1):163.

4. Al-Bedah AM, Ali Gl, Abushanab TS, Qureshi NA. Tui Na (or Tuina) massage: a minireview of pertinent literature, 1970-2017. J Complement Altern Med Res. 2017;3(1):1-14.

5. McCarthy M. Palpatory literacy, Chinese therapeutic bodywork (Tui Na) and the remediation of head, neck and shoulder pain. J Bodyw Mov Ther. 2003;7(4):262-77.

6. Field T. Massage therapy research review. Complemen Ther Clin Pract. 2016;24:19-31.

7. Ernst E. The safety of massage therapy. Rheumatology. 2003;42:1101-6.

8. Field T, Diego M, Hernandez-Reif M. Massage therapy research. Dev Rev. 2007;27:75-89.

9. Candy B, Armstrong M, Flemming K, Kupeli N, Stone P, Vickerstaff V, et al. The effectiveness of aromatherapy, massage and reflexology in people with palliative care needs: a systematic review. Palliat Med. 2020;34(2):179-94.

10. Zhang XT, Hu YY, Zhang XY, Chen HQ, Wang XL, Li XY, et al. An overview of systematic reviews on Tuina from 2013 to 2017. Chin J Evid Based Med. 2019;19(3):361-7 (Article in Chinese).

11. Schulz KF, Altman DG, Moher D, CONSORT Group. CONSORT 2010 statement: updated guidelines for reporting parallel group randomized trials. Ann Intern Med. 2010;152(11):726-32.

12. Boutron I, Altman DG, Moher D, Schulz KF, Ravaud P, CONSORT NPT Group. CONSORT statement for randomized trials of nonpharmacologic treatments: a 2017 update and a CONSORT extension for nonpharmacologic trial abstracts. Ann Intern Med. 2017;167(1):40-7.

13. Cheng CW, Wu TX, Shang HC, Li YP, Altman DG, Moher D, et al. CONSORTCHM Formulas 2017 Group. CONSORT Extension for Chinese Herbal Medicine Formulas 2017: Recommendations, Explanation, and Elaboration. Ann Intern Med. 2017;167(2):112-121.

14. MacPherson $\mathrm{H}$, Altman DG, Hammerschlag $R$, Youping L, Taixiang W, White $A$, et al. Revised standards for reporting interventions in clinical trials of acupuncture (STRICTA): extending the CONSORT statement. PLOS Med. 2010;7(6):e1000261.

15. Cheng CW, Fu SF, Zhou QH, Wu TX, Shang HC, Tang XD, et al. Extending the CONSORT statement to moxibustion. J Integr Med. 2013;11:54-63.

16. Zhang X, Tian R, Lam WC, Duan YT, Liu F, Zhao C, et al. Standards for reporting interventions in clinical trials of cupping (STRICTOC): extending the CONSORT statement. Chin Med. 2020;15:10.

17. Turner L, Shamseer L, Altman DG, Schulz KF, Moher D. Does use of the CONSORT Statement impact the completeness of reporting of randomised controlled trials published in medical journals? A Cochrane review Syst Rev. 2012;1:60. 
18. Kim KH, Kang JW, Lee MS, Lee JD. Assessment of the quality of reporting in randomised controlled trials of acupuncture in the Korean literature using the CONSORT statement and STRICTA guidelines. BMJ Open. 2014;4:e005068.

19. Li J, Gao W, Punja S, Ma B, Vohra S, Duan N, et al. Reporting quality of N-of-1 trials published between 1985 and 2013: a systematic review. J Clin Epidemiol. 2016;76:57-64.

20. Song TJ, Leng HF, Zhong LLD, Wu TX, Bian ZX. CONSORT in China: past development and future direction. Trials. 2015;16:243.

21. Liu YL, Zhang R, Huang J, Zhao X, Liu DL, Sun WT, et al. Reporting quality of systematic reviews/meta-analyses of acupuncture. PLOS ONE. 2014;9(11):e113172

22. Institute of Scientific and Technical Information of China. 2019 China Science and Technology Core Journals Catalog (Natural Science Volume). Published in 19 Nov 2019, pp:1-40. https://www.bjtjzx.com/ywpx/xiazai/ 2020/2019\%E5\%B9\%B4\%E4\%B8\%AD\%E5\%9B\%BD\%E7\%A7\%91\%E6\% 8A\%80\%E6\%A0\%B8\%E5\%BF\%83\%E6\%9C\%9F\%E5\%88\%8A\%E7\%9B\% AE\%E5\%BD\%95\%EF\%BC\%88\%E8\%87\%AA\%E7\%84\%B6\%E7\%A7\%91\% E5\%AD\%A6\%E5\%8D\%B7\%EF\%BC\%89.pdf (In Chinese). Accessed 11 Dec 2020.

23. Liu JP, Han M, Li XX, Mu YJ, Lewith G, Wang YY, et al. Prospective registration, bias risk and outcome-reporting bias in randomised clinical trials of traditional Chinese medicine: an empirical methodological study. BMJ Open. 2013;3:e002968.

24. Ferrarello F, Viligiardi M, Bari MD. Ethics reporting practices in randomized controlled trials of physical therapy interventions after stroke. Arch Physiother. 2018;8:8.

25. Wang G, Mao B, Xiong ZY, Fan T, Chen XD, Wang $L$, et al. The quality of reporting of randomized controlled trials of traditional Chinese medicine: a survey of 13 randomly selected journals from mainland China. Clin Ther 2007;29:1456-67.

26. Qian GF, Zeng JC, Lu LM, Pei WY, Liu K, Luo ZK, et al. Evaluation of reporting quality in randomised controlled trials of acupuncture for acute herpes zoster by the CONSORT statement and STRICTA guidelines. Evid Based Complement Alternat Med. 2020;2020:4308380.

27. Moher D, Hopewell S, Schulz KF, Altman DG. Resources for authors of reports of randomized trials: harnessing the wisdom of authors, editors, and readers. Trials. 2011;12:98.

28. Tian R, Zhao C, Cheng CW, Lam WC, Yang Z, Wu TX, et al. Constructing the Chinese medicine clinical curative effects research report system. Chin J Evid Based Med. 2018;18(7):651-3 (Article in Chinese).

29. Yu J, Li X, Li Y, Sun X. Quality of reporting in surgical randomized clinical trials. Br J Surg. 2017;104:296-303.

30. Liu K, Zeng JC, Pei WY, Chen SY, Luo ZK, Lu LM, et al. Assessing the reporting quality in randomized controlled trials of acupuncture for postherpetic neuralgia using the CONSORT statement and STRICTA guidelines. J Pain Res. 2019;12:2359-70.

31. Liu SW, Zhao N, Zhang W, Li HN, Luo XF, Wang JG. Introduction about the theoretical and methodological system of Jingu infantile tuina school. Chin J Tradit Chin Med Pharm. 2019;34(11):5024-6 (Article in Chinese).

32. Sun SQ, Li HN, Liu SW, Han YH, Dong H. Comparative study of the main abdominal Tuina sect in China. J Tianjin Univ Tradit Chin Med. 2018;37(3):183-7 (Article in Chinese).

33. Wang JL, Sun TT, Lin YW, Lu R, Fang JY. Methodological reporting of randomized controlled trials in major hepato-gastroenterology journals in 2008 and 1998: a comparative study. BMC Medical Res Methodol. 2011:11:110.

34. Enhancing the QUAlity and Transparency Of health Research (EQUAOR) Network. https://www.equator-network.org/library/reporting-guidelinesunder-development/reporting-guidelines-under-development-for-clini cal-trials/\#STRICTOTM. Accessed 11 July 2020.

\section{Publisher's Note}

Springer Nature remains neutral with regard to jurisdictional claims in published maps and institutional affiliations.
Ready to submit your research? Choose BMC and benefit from:

- fast, convenient online submission

- thorough peer review by experienced researchers in your field

- rapid publication on acceptance

- support for research data, including large and complex data types

- gold Open Access which fosters wider collaboration and increased citations

- maximum visibility for your research: over 100M website views per year

At BMC, research is always in progress.

Learn more biomedcentral.com/submissions 